\title{
2-(3-Pyridyl)thiazolidine-4-carboxamides. 1. Novel Orally Active Antagonists of Platelet-Activating Factor (PAF) ${ }^{1)}$
}

\author{
Hitoshi Nagaoka, ${ }^{2)}$ Hiromu Hara, Takeshi Suzuki, Takumi TaKahashi, Makoto Takeuchi, \\ Akira Matsuhisa, Munetoshi Saito, Toshimitsu Yamada, Kenichi Tomioka, and \\ Toshiyasu MASE*
}

Institute for Drug Discovery Research, Yamanouchi Pharmaceutical Co., Ltd., 21 Miyukigaoka, Tsukuba, Ibaraki 305, Japan. Received May 26, 1997; accepted June 11, 1997

In a search for novel platelet-activating factor (PAF) antagonists, we found that 1-(3-phenylpropyl)-4-[2-(3pyridyl)thiazolidine-4-carbonyl]piperazine (3x) showed in vitro and in vivo PAF-antagonistic activities. Introduction of functional groups at the benzylic methylene carbon of $3 x$ afforded some compounds with more potent PAFantagonistic activity than 3x. Among them 1-(3-methyl-3-phenylbutyl)-4-[2-(3-pyridyl)thiazolidine-4-carbonyl]piperazine fumarate (3al, YM264) was found to be one of the most potent PAF antagonists.

Key words [2-(3-pyridyl)thiazolidine-4-yl]carboxamide; PAF antagonist; structure-activity relationship; YM264

Platelet activating factor (PAF) is an ether-linked phospholipid mediator, which is released from a variety of cell types including neutrophils, eosinophils, macrophages, platelets and endothelial cells. ${ }^{3)}$ PAF has a wide spectrum of biological actions, such as induction of platelet aggregation, increase in vascular permeability, bronchoconstriction, systemic hypotension, and cardiac and renal dysfunction. ${ }^{4-8)}$ Because of its various actions, PAF has been suggested to play an important role in the pathogenesis of asthma, shock, glomerulonephritis and other diseases. ${ }^{9)}$ The development of PAF antagonists therefore may offer a novel approach to the prevention and therapy of these diseases. For this reason, a search for novel PAF antagonists is under way and several compounds including PAF analogues (CV-3988, CV6209), thienodiazepines (WEB-2086, Y-24180, E-6123) and other compounds have already been reported. ${ }^{10)}$ Some of them are under clinical trial.

In a search for potent and orally active PAF antagonists, we tried a chemical file screening approach and found that 2-(3-pyridyl)-2,3-dihydrobenzothiazole (1) is almost equipotent to RP48740 (2), ${ }^{11)}$ a PAF antagonist, in inhibiting PAF-induced platelet aggregation $\left(\mathrm{IC}_{50}=\right.$ $15 \mu \mathrm{M})$. We were interested in the similarity between 1 and 2 in chemical structure and prepared a variety of arylsubstituted heterocyclic compounds, which we tested for PAF-antagonistic activity. Among them, pyridyl thiazolidinecarboxamide (3a) was found to be more potent than 1. Then we focused our attention on pyridyl thiazolidinecarboxamides 3 and prepared a number of their derivatives for activity testing. In this paper, we describe the synthesis and structure-activity relationship of a novel series of pyridyl thiazolidinecarboxamides as PAF antagonists.

\section{Synthesis}

The 3-pyridylthiazolidinecarboxamides (3) were prepared as described below (Chart 2). The 3-pyridylthiazolidinecarboxylic acid (4), prepared from 3-pyridylaldehyde and $\mathrm{L}$-cysteine, was treated with various amines in the presence of dicyclohexylcarbodiimide (DCC) and 1-hydroxybenzotriazole (HOBT) (method A), or diphenylphosphorylazide (DPPA) (method B) to give the amides (3). In some cases,
4 was converted to tert-butylcarbamate (5) and then coupled with amines to give $\mathbf{6}$. Finally, $\mathbf{6}$ was treated with trifluoroacetic acid (TFA) to furnish the amide (3) (method C). Compound $\mathbf{3 x}$ could also be prepared by the coupling of anhydride (7) and an amine (method D). The anhydride (7) was easily prepared as stable crystals from 5 by the treatment with $(\mathrm{COCl})_{2}$ and $\mathrm{Et}_{3} \mathrm{~N}$.

It is well-known that 2,4-disubstituted thiazolidine isomerizes freely between the cis and trans diastereomers in solution and exists as equilibrium mixture. ${ }^{12)}$ NMR spectra revealed that all of the compounds prepared here exist as an about 1 to 1 mixture of cis and trans isomers in solution. Configurations at C-4 are considered to be $R$ because the compounds were derived from L-cysteine.

\section{Pharmacological Activity and Discussion}

The compounds were tested for PAF-antagonistic activity by measuring the inhibition of PAF-induced platelet aggregation using rabbit platelet-rich plasma (PRP).

Compound 3a antagonized PAF-induced platelet aggregation with an $\mathrm{IC}_{50}$ of $1.5 \mu \mathrm{M}$. Deletion of the alkylsulfide group (3b) reduced the activity, while deletion of the ester group (3c) retained the activity. Simple alkyl amides (3d, 3e) retained the potency. Anilide (3f) and benzylamide (3g) derivatives also exhibited PAF-antagonist activity and the introduction of a methoxy group (3h) in the phenyl ring of $\mathbf{3 g}$ improved the activity. The introduction of hydrophobic groups such as phenylalkoxy $(3 \mathbf{i}-\mathbf{m})$ and branched alkoxy (3n) groups in the phenyl ring of $3 \mathrm{~g}$ caused further improvement of the antagonistic

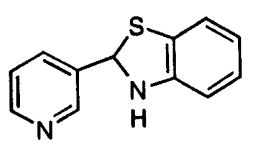

1

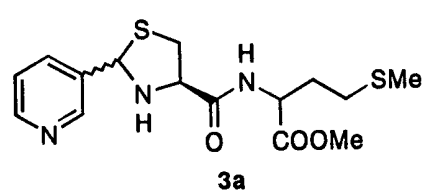

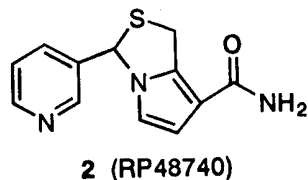

2 (RP48740)

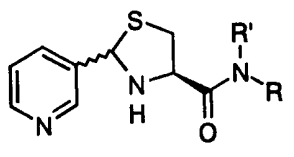

3
Chart 1

(C) 1997 Pharmaceutical Society of Japan 


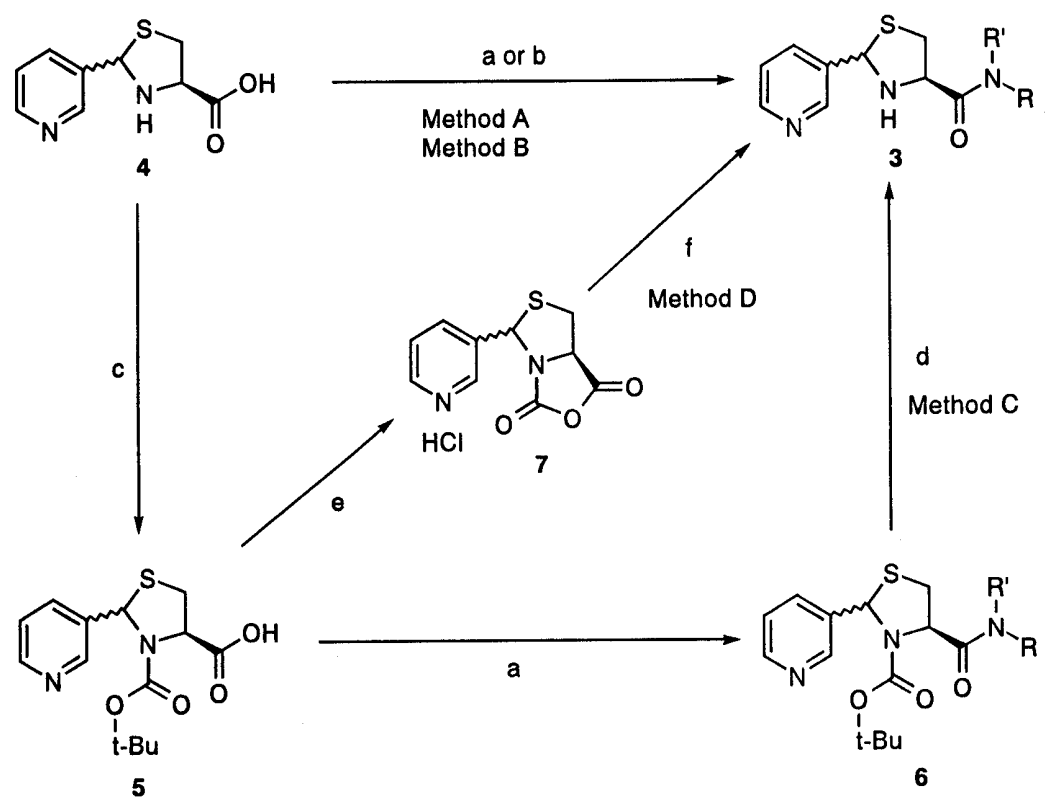

(a) $\mathrm{RNH}_{2}, \mathrm{DCC}, \mathrm{HOBT} / \mathrm{THF}$ or DMF; (b) $\mathrm{RNH}_{2}, \mathrm{DPPA}, \mathrm{Et}_{3} \mathrm{~N} / \mathrm{DMF}$;

(c) (t-BuOCO) ${ }_{2} \mathrm{O}, \mathrm{NaOH} /$ dioxane, $\mathrm{H}_{2} \mathrm{O}$; (d) TFA; (e) $(\mathrm{COCl})_{2}, \mathrm{DMF}, \mathrm{CH}_{2} \mathrm{Cl}_{2}$;

(f) $\mathrm{RNH}_{2}, \mathrm{DMSO}$

Chart 2

Table 1. Physicochemical Data and PAF-antagonist Activities for Thiazolidinecarboxamides<smiles>[R]N([R])C(=O)C1CS[C@@H](c2cccnc2)N1</smiles>

\begin{tabular}{|c|c|c|c|c|c|c|c|c|c|c|c|}
\hline \multirow{2}{*}{$\begin{array}{l}\text { Compd. } \\
\text { No. }\end{array}$} & \multirow{2}{*}{$\mathrm{R}$} & \multirow{2}{*}{$\mathrm{R}^{\prime}$} & \multirow{2}{*}{ Salt } & \multirow{2}{*}{ Method } & \multirow{2}{*}{$\begin{array}{l}\mathrm{mp} \\
\left({ }^{\circ} \mathrm{C}\right)\end{array}$} & \multirow{2}{*}{ Formula } & \multicolumn{4}{|c|}{$\begin{array}{l}\text { Analysis (\%) } \\
\text { Calcd (Found) }\end{array}$} & \multirow{2}{*}{$\begin{array}{c}\text { Platelet } \\
\text { aggregation } \\
\text { inhibition } \\
\mathrm{IC}_{50}(\mu \mathrm{M})\end{array}$} \\
\hline & & & & & & & $\mathrm{C}$ & $\mathrm{H}$ & $\mathrm{N}$ & $\mathrm{S}$ & \\
\hline $3 \mathbf{a}$ & $\mathrm{CH}\left(\mathrm{COOCH}_{3}\right) \mathrm{CH}_{2} \mathrm{CH}_{2} \mathrm{SCH}_{3}$ & $\mathrm{H}$ & - & $\mathrm{C}$ & Oil & $\mathrm{C}_{15} \mathrm{H}_{21} \mathrm{~N}_{3} \mathrm{O}_{3} \mathrm{~S}_{2}$ & $\begin{array}{r}50.68 \\
(50.39\end{array}$ & $\begin{array}{l}5.95 \\
5.93\end{array}$ & $\begin{array}{l}11.82 \\
11.60)\end{array}$ & & 1.5 \\
\hline 3b & $\mathrm{CH}_{2} \mathrm{COOCH}_{3}$ & $\mathrm{H}$ & - & $\mathrm{C}$ & Oil & $\begin{array}{l}\mathrm{C}_{12} \mathrm{H}_{15} \mathrm{~N}_{3} \mathrm{O}_{3} \mathrm{~S} \\
\cdot 0.3 \mathrm{H}_{2} \mathrm{O}\end{array}$ & $\begin{array}{r}50.27 \\
(50.18\end{array}$ & $\begin{array}{l}5.48 \\
5.36\end{array}$ & $\begin{array}{l}14.65 \\
14.31\end{array}$ & $\begin{array}{l}11.18 \\
11.19)\end{array}$ & 14 \\
\hline $3 \mathbf{c}$ & $\left(\mathrm{CH}_{2}\right)_{3} \mathrm{SCH}_{3}$ & $\mathrm{H}$ & - & $\mathrm{C}$ & Oil & $\mathrm{C}_{13} \mathrm{H}_{21} \mathrm{Cl}_{2} \mathrm{~N}_{3} \mathrm{OS}_{2}$ & $\begin{array}{r}52.55 \\
(52.49\end{array}$ & $\begin{array}{l}6.26 \\
6.44\end{array}$ & $\begin{array}{l}14.11 \\
14.11\end{array}$ & $\begin{array}{l}21.62 \\
21.56)\end{array}$ & 1.6 \\
\hline 3d & $\left(\mathrm{CH}_{2}\right)_{6} \mathrm{CH}_{3}$ & $\mathrm{H}$ & - & $\mathrm{C}$ & 79 & $\mathrm{C}_{16} \mathrm{H}_{25} \mathrm{~N}_{3} \mathrm{OS}$ & $\begin{array}{r}62.50 \\
62.50\end{array}$ & $\begin{array}{l}8.20 \\
8.22\end{array}$ & $\begin{array}{l}13.67 \\
13.56\end{array}$ & $\begin{array}{l}10.43 \\
10.23)\end{array}$ & 1.5 \\
\hline $3 \mathbf{e}$ & $\left(\mathrm{CH}_{2}\right)_{9} \mathrm{CH}_{3}$ & $\mathrm{H}$ & - & A & 88 & $\mathrm{C}_{19} \mathrm{H}_{31} \mathrm{~N}_{3} \mathrm{OS}$ & $\begin{array}{r}65.48 \\
(65.16\end{array}$ & $\begin{array}{l}8.68 \\
8.80\end{array}$ & $\begin{array}{l}12.06 \\
11.91\end{array}$ & $\begin{array}{l}9.20 \\
9.04)\end{array}$ & 3.9 \\
\hline $3 f$ & $\mathrm{C}_{6} \mathrm{H}_{5}$ & $\mathrm{H}$ & - & A & Oil & $\begin{array}{l}\mathrm{C}_{15} \mathrm{H}_{15} \mathrm{~N}_{3} \mathrm{OS} \\
\cdot 0.2 \mathrm{H}_{2} \mathrm{O}\end{array}$ & $\begin{array}{r}62.35 \\
62.74\end{array}$ & $\begin{array}{l}5.37 \\
7.30\end{array}$ & $\begin{array}{l}14.54 \\
14.52\end{array}$ & $\begin{array}{l}11.10 \\
11.25)\end{array}$ & 2.5 \\
\hline $3 \mathrm{~g}$ & $\mathrm{CH}_{2} \mathrm{C}_{6} \mathrm{H}_{5}$ & $\mathrm{H}$ & - & A & Oil & $\begin{array}{l}\mathrm{C}_{16} \mathrm{H}_{17} \mathrm{~N}_{3} \mathrm{OS} \\
\cdot 0.2 \mathrm{H}_{2} \mathrm{O}\end{array}$ & $\begin{array}{r}63.42 \\
(63.55\end{array}$ & $\begin{array}{l}5.79 \\
5.83\end{array}$ & $\begin{array}{l}13.87 \\
13.85\end{array}$ & $\begin{array}{l}10.58 \\
10.34)\end{array}$ & 3.7 \\
\hline $3 \mathbf{h}$ & $\mathrm{CH}_{2} \mathrm{C}_{6} \mathrm{H}_{4}-4-\left(\mathrm{OCH}_{3}\right)$ & $\mathrm{H}$ & - & A & 103 & $\mathrm{C}_{17} \mathrm{H}_{19} \mathrm{~N}_{3} \mathrm{O}_{2} \mathrm{~S}$ & $\begin{array}{r}61.98 \\
(61.99\end{array}$ & $\begin{array}{l}5.81 \\
5.81\end{array}$ & $\begin{array}{l}12.76 \\
12.67\end{array}$ & $\begin{array}{l}9.73 \\
9.94)\end{array}$ & 0.90 \\
\hline $3 \mathbf{i}$ & $\mathrm{CH}_{2} \mathrm{C}_{6} \mathrm{H}_{4}-4-\left[\mathrm{O}\left(\mathrm{CH}_{2}\right)_{4} \mathrm{C}_{6} \mathrm{H}_{5}\right]$ & $\mathrm{H}$ & $2 \mathrm{HCl}$ & A & 135 & $\begin{array}{l}\mathrm{C}_{26} \mathrm{H}_{29} \mathrm{~N}_{3} \mathrm{O}_{2} \mathrm{~S} \\
\cdot 0.2 \mathrm{H}_{2} \mathrm{O} \cdot 2 \mathrm{HCl}\end{array}$ & $\begin{array}{r}59.58 \\
(59.58\end{array}$ & $\begin{array}{l}6.04 \\
6.02\end{array}$ & $\begin{array}{l}8.02 \\
7.96\end{array}$ & $\begin{array}{l}6.12 \\
6.23)\end{array}$ & 0.43 \\
\hline $3 \mathbf{j}$ & $\mathrm{CH}_{2} \mathrm{C}_{6} \mathrm{H}_{4}-4-\left(\mathrm{OCH}_{2} \mathrm{C}_{6} \mathrm{H}_{5}\right)$ & $\mathrm{H}$ & - & B & 140 & $\mathrm{C}_{23} \mathrm{H}_{23} \mathrm{~N}_{3} \mathrm{O}_{2} \mathrm{~S}$ & $\begin{array}{r}68.12 \\
(68.23\end{array}$ & $\begin{array}{l}5.72 \\
6.74\end{array}$ & $\begin{array}{l}10.36 \\
10.31\end{array}$ & $\begin{array}{l}7.91 \\
8.01)\end{array}$ & 0.38 \\
\hline $3 \mathbf{k}$ & $\mathrm{CH}_{2} \mathrm{C}_{6} \mathrm{H}_{6}-4-\left[\mathrm{O}\left(\mathrm{CH}_{2}\right)_{2} \mathrm{C}_{6} \mathrm{H}_{5}\right]$ & $\mathrm{H}$ & - & B & 116 & $\mathrm{C}_{24} \mathrm{H}_{25} \mathrm{~N}_{3} \mathrm{O}_{2} \mathrm{~S}$ & $\begin{array}{r}68.71 \\
(68.56\end{array}$ & $\begin{array}{l}6.01 \\
6.01\end{array}$ & $\begin{array}{r}10.02 \\
9.94\end{array}$ & $\begin{array}{l}7.64 \\
7.63)\end{array}$ & 0.15 \\
\hline 3I & $\mathrm{CH}_{2} \mathrm{C}_{6} \mathrm{H}_{4}-4-\left[\mathrm{O}\left(\mathrm{CH}_{2}\right)_{3} \mathrm{C}_{6} \mathrm{H}_{5}\right]$ & $\mathrm{H}$ & $2 \mathrm{HCl}$ & A & 116 & $\begin{array}{l}\mathrm{C}_{25} \mathrm{H}_{27} \mathrm{~N}_{3} \mathrm{O}_{2} \mathrm{~S} \\
\cdot 2 \mathrm{HCl}\end{array}$ & $\begin{array}{r}59.28 \\
(58.95\end{array}$ & $\begin{array}{l}5.77 \\
5.74\end{array}$ & $\begin{array}{l}8.30 \\
8.21\end{array}$ & $\begin{array}{l}6.33 \\
6.36)\end{array}$ & 0.39 \\
\hline $3 m$ & $\mathrm{CH}_{2} \mathrm{C}_{6} \mathrm{H}_{4}-4-\left[\mathrm{O}\left(\mathrm{CH}_{2}\right)_{5} \mathrm{C}_{6} \mathrm{H}_{5}\right]$ & $\mathrm{H}$ & - & B & 83 & $\mathrm{C}_{27} \mathrm{H}_{31} \mathrm{~N}_{3} \mathrm{O}_{2} \mathrm{~S}$ & $\begin{array}{r}70.25 \\
(70.33\end{array}$ & $\begin{array}{l}6.77 \\
6.81\end{array}$ & $\begin{array}{l}9.10 \\
9.06\end{array}$ & $\begin{array}{l}6.95 \\
6.84)\end{array}$ & 0.58 \\
\hline $3 \mathbf{n}$ & $\begin{array}{c}\mathrm{CH}_{2} \mathrm{C}_{6} \mathrm{H}_{4}-4- \\
{\left[\mathrm{O}\left(\mathrm{CH}_{2}\right)_{2} \mathrm{CH}\left(\mathrm{CH}_{3}\right)_{2}\right]}\end{array}$ & $\mathrm{H}$ & $2 \mathrm{HCl}$ & A & 128 & $\begin{array}{l}\mathrm{C}_{21} \mathrm{H}_{27} \mathrm{~N}_{3} \mathrm{O}_{2} \mathrm{~S} \\
\cdot 2 \mathrm{HCl} \cdot 0.4 \mathrm{H}_{2} \mathrm{O}\end{array}$ & $\begin{array}{r}54.17 \\
(54.23\end{array}$ & $\begin{array}{l}6.45 \\
6.37\end{array}$ & $\begin{array}{l}9.02 \\
8.96\end{array}$ & $\begin{array}{l}6.89 \\
7.00)\end{array}$ & 0.24 \\
\hline 30 & $\begin{array}{l}\mathrm{CH}_{2} \mathrm{C}_{6} \mathrm{H}_{5} \\
\text { WEB-2086 }\end{array}$ & $\mathrm{Me}$ & $2 \mathrm{HCl}$ & A & 110 & $\begin{array}{l}\mathrm{C}_{17} \mathrm{H}_{19} \mathrm{~N}_{3} \mathrm{OS} \\
\cdot 2 \mathrm{HCl} \cdot \mathrm{H}_{2} \mathrm{O}\end{array}$ & $\begin{array}{r}50.50 \\
(50.63\end{array}$ & $\begin{array}{l}5.73 \\
5.60\end{array}$ & $\begin{array}{l}10.39 \\
10.43\end{array}$ & $\begin{array}{l}7.93 \\
7.98)\end{array}$ & 0.79 \\
\hline
\end{tabular}


activity. The tertiary amide (3o) was about four times more potent than the corresponding secondary amide $(\mathbf{3 g})$. From these results, it was revealed that hydrophobic groups were favorable as $N$-substituents for PAF-antagonistic activity (Table 1).

Based on these results, we focused on cyclic amine amides as a hydrophobic moiety and tertiary amides. The results are shown in Table 2. Pyrrolidine (3p) and morpholine (3r) amides showed weaker activity than 3a. Piperidine (3q) and 4-carboethoxypiperazine (3s) amides retained the activity. Methylpiperazide (3t) showed reduced activity while phenyl piperazide (3u) was almost as potent as 3s. Furthermore, the introduction of more hydrophobic groups such as phenylalkyl $(\mathbf{3 v}-\mathbf{3 z})$ and long chain alkyl (3aa-3ae) groups at the 4-position of the piperazine ring of $3 \mathbf{u}$ improved the antagonistic activity. Among these compounds, 3x, 3z, 3ac and 3ad exhibited strong PAF-antagonist activities. On the other hand, 4-substituted piperidides (3af, 3ag) were much less potent than the corresponding piperazides $(\mathbf{3 w}, \mathbf{3 x})$ and the $S$-isomer of 3x (S-3x: prepared from D-cysteine) showed less activity $(70 \%$ inhibition at $100 \mu \mathrm{M})$. The piperidyl amide (3ah) also showed weak antagonistic activity.

Based on these results, benzyl amides (3l, 3n) and piperazyl amides (3x, 3z, 3ac, 3ad) were selected for further evaluation. The results are shown in Table 3 .

In the in vitro study, most of the compounds inhibited PAF-induced aggregation of human platelets. It is interesting that $\mathbf{3 z}$ was ten times less potent than $\mathbf{3 x}$ in human platelets, though they were almost equipotent in rabbit

Table 2. Physicochemical Data and PAF-Antagonist Activities for Thiazolidinecarboxamides

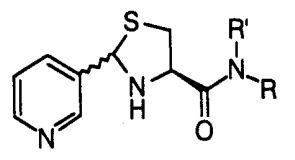

\begin{tabular}{|c|c|c|c|c|c|c|c|c|c|c|}
\hline \multirow{2}{*}{$\begin{array}{l}\text { Compd. } \\
\text { No. }\end{array}$} & \multirow{2}{*}{$\mathrm{NRR}^{\prime}$} & \multirow{2}{*}{ Salt } & \multirow{2}{*}{ Method } & \multirow{2}{*}{$\begin{array}{l}\mathrm{mp} \\
\left({ }^{\circ} \mathrm{C}\right)\end{array}$} & \multirow{2}{*}{ Formula } & \multicolumn{4}{|c|}{$\begin{array}{l}\text { Analysis (\%) } \\
\text { Calcd (Found) }\end{array}$} & \multirow{2}{*}{$\begin{array}{c}\text { Platelet } \\
\text { aggregation } \\
\text { inhibition } \\
\mathrm{IC}_{50}(\mu \mathrm{M})\end{array}$} \\
\hline & & & & & & $\mathrm{C}$ & $\mathrm{H}$ & $\mathrm{N}$ & S & \\
\hline $3 p$ & 1-pyrrolidinyl & $2 \mathrm{HCl}$ & A & 136 & $\begin{array}{l}\mathrm{C}_{13} \mathrm{H}_{17} \mathrm{~N}_{3} \mathrm{OS} \cdot 2 \mathrm{HCl} \\
\cdot \mathrm{H}_{2} \mathrm{O} \cdot 0.3 \text { dioxane }\end{array}$ & $\begin{array}{r}44.80 \\
(44.62\end{array}$ & $\begin{array}{l}6.19 \\
5.95\end{array}$ & $\begin{array}{l}11.04 \\
10.89\end{array}$ & $\begin{array}{l}8.42 \\
8.65)\end{array}$ & 21 \\
\hline $3 q$ & 1-piperidinyl & $2 \mathrm{HCl}$ & A & 172 & $\begin{array}{l}\mathrm{C}_{14} \mathrm{H}_{19} \mathrm{~N}_{3} \mathrm{OS} \cdot 2 \mathrm{HCl} \\
0.3 \mathrm{H}_{2} \mathrm{O}\end{array}$ & $\begin{array}{r}47.27 \\
(47.36\end{array}$ & $\begin{array}{l}6.12 \\
6.03\end{array}$ & $\begin{array}{l}11.81 \\
11.75\end{array}$ & $\begin{array}{l}9.01 \\
9.01)\end{array}$ & 3.0 \\
\hline $3 r$ & 4-morpholinyl & $2 \mathrm{HCl}$ & A & 143 & $\begin{array}{l}\mathrm{C}_{13} \mathrm{H}_{17} \mathrm{~N}_{3} \mathrm{O}_{2} \mathrm{~S} \cdot 2 \mathrm{HCl} \\
0.3 \mathrm{H}_{2} \mathrm{O}\end{array}$ & $\begin{array}{r}43.65 \\
(43.79\end{array}$ & $\begin{array}{l}5.52 \\
5.22\end{array}$ & $\begin{array}{l}11.75 \\
11.66\end{array}$ & $\begin{array}{l}8.96 \\
9.09)\end{array}$ & 14 \\
\hline $3 \mathrm{~s}$ & $\mathrm{~N}\left(\mathrm{CH}_{2} \mathrm{CH}_{2}\right)_{2} \mathrm{NCOOEt}$ & $2 \mathrm{HCl}$ & A & Oil & $\mathrm{C}_{16} \mathrm{H}_{22} \mathrm{~N}_{4} \mathrm{O}_{3} \mathrm{~S} \cdot 2 \mathrm{HCl}$ & $\begin{array}{r}45.39 \\
(45.12\end{array}$ & $\begin{array}{l}5.71 \\
5.52\end{array}$ & $\begin{array}{l}13.23 \\
13.02)\end{array}$ & & 1.1 \\
\hline $3 t$ & $\mathrm{~N}\left(\mathrm{CH}_{2} \mathrm{CH}_{2}\right)_{2} \mathrm{NMe}$ & $3 \mathrm{HCl}$ & $\mathrm{C}$ & 182 & $\begin{array}{l}\mathrm{C}_{14} \mathrm{H}_{20} \mathrm{~N}_{4} \mathrm{OS} \cdot 3 \mathrm{HCl} \\
\cdot 0.5 \mathrm{H}_{2} \mathrm{O}\end{array}$ & $\begin{array}{r}40.93 \\
(41.02\end{array}$ & $\begin{array}{l}5.89 \\
5.89\end{array}$ & $\begin{array}{l}13.64 \\
13.55\end{array}$ & $\begin{array}{l}7.81 \\
7.61)\end{array}$ & 7.7 \\
\hline $3 \mathbf{u}$ & $\mathrm{N}\left(\mathrm{CH}_{2} \mathrm{CH}_{2}\right)_{2} \mathrm{NPh}$ & $3 \mathrm{HCl}$ & A & 169 & $\begin{array}{l}\mathrm{C}_{19} \mathrm{H}_{22} \mathrm{~N}_{4} \mathrm{OS} \cdot 3 \mathrm{HCl} \\
\cdot 0.3 \mathrm{H}_{2} \mathrm{O}\end{array}$ & $\begin{array}{r}48.63 \\
(48.73\end{array}$ & $\begin{array}{l}5.50 \\
5.67\end{array}$ & $\begin{array}{l}11.94 \\
11.71\end{array}$ & $\begin{array}{l}6.83 \\
6.70)\end{array}$ & 0.77 \\
\hline $3 v$ & $\mathrm{~N}\left(\mathrm{CH}_{2} \mathrm{CH}_{2}\right)_{2} \mathrm{NCH}_{2} \mathrm{Ph}$ & $3 \mathrm{HCl}$ & A & 165 & $\begin{array}{l}\mathrm{C}_{20} \mathrm{H}_{24} \mathrm{~N}_{4} \mathrm{OS} \cdot 3 \mathrm{HCl} \\
\cdot 0.5 \mathrm{H}_{2} \mathrm{O}\end{array}$ & $\begin{array}{r}49.34 \\
(49.29\end{array}$ & $\begin{array}{l}5.80 \\
5.87\end{array}$ & $\begin{array}{l}11.51 \\
11.46\end{array}$ & $\begin{array}{l}6.59 \\
6.83)\end{array}$ & 0.45 \\
\hline $3 w$ & $\mathrm{~N}\left(\mathrm{CH}_{2} \mathrm{CH}_{2}\right)_{2} \mathrm{~N}\left(\mathrm{CH}_{2}\right)_{2} \mathrm{Ph}$ & $3 \mathrm{HCl}$ & A & 155 & $\begin{array}{l}\mathrm{C}_{21} \mathrm{H}_{25} \mathrm{~N}_{4} \mathrm{OS} \cdot 3 \mathrm{HCl} \\
\cdot 0.4 \mathrm{H}_{2} \mathrm{O}\end{array}$ & $\begin{array}{r}50.64 \\
(50.74\end{array}$ & $\begin{array}{l}5.83 \\
6.11\end{array}$ & $\begin{array}{l}11.25 \\
11.21\end{array}$ & $\begin{array}{l}6.44 \\
6.44)\end{array}$ & 0.22 \\
\hline $3 \mathbf{x}$ & $\mathrm{N}\left(\mathrm{CH}_{2} \mathrm{CH}_{2}\right)_{2} \mathrm{~N}\left(\mathrm{CH}_{2}\right)_{3} \mathrm{Ph}$ & Fumarate & $A, D$ & 175 & $\mathrm{C}_{26} \mathrm{H}_{32} \mathrm{~N}_{4} \mathrm{O}_{5} \mathrm{~S}$ & $\begin{array}{r}60.92 \\
(60.62\end{array}$ & $\begin{array}{l}6.29 \\
6.25\end{array}$ & $\begin{array}{l}10.62 \\
10.79\end{array}$ & $\begin{array}{l}6.26 \\
6.17)\end{array}$ & 0.071 \\
\hline$(S)-\mathbf{3 x}$ & $\mathrm{N}\left(\mathrm{CH}_{2} \mathrm{CH}_{2}\right)_{2} \mathrm{~N}\left(\mathrm{CH}_{2}\right)_{3} \mathrm{Ph}$ & Fumarate & A & 191 & $\mathrm{C}_{26} \mathrm{H}_{32} \mathrm{~N}_{4} \mathrm{O}_{5} \mathrm{~S}$ & $\begin{array}{r}60.92 \\
(60.68\end{array}$ & $\begin{array}{l}6.29 \\
6.15\end{array}$ & $\begin{array}{l}10.62 \\
10.86\end{array}$ & $\begin{array}{l}6.26 \\
6.13)\end{array}$ & $\begin{array}{l}70 \% \text { inhibn. } \\
\text { at } 100 \mu \mathrm{M}\end{array}$ \\
\hline $3 y$ & $\mathrm{~N}\left(\mathrm{CH}_{2} \mathrm{CH}_{2}\right)_{2} \mathrm{~N}\left(\mathrm{CH}_{2}\right)_{4} \mathrm{Ph}$ & $3 \mathrm{HCl}$ & A & 157 & $\begin{array}{l}\mathrm{C}_{23} \mathrm{H}_{29} \mathrm{~N}_{4} \mathrm{OS} \cdot 3 \mathrm{HCl} \\
\cdot \mathrm{H}_{2} \mathrm{O}\end{array}$ & $\begin{array}{r}51.45 \\
(51.32\end{array}$ & $\begin{array}{l}6.38 \\
6.41\end{array}$ & $\begin{array}{l}10.43 \\
10.43\end{array}$ & $\begin{array}{l}5.97 \\
5.98)\end{array}$ & 0.12 \\
\hline $3 z$ & $\mathrm{~N}\left(\mathrm{CH}_{2} \mathrm{CH}_{2}\right)_{2} \mathrm{~N}\left(\mathrm{CH}_{2}\right)_{5} \mathrm{Ph}$ & $3 \mathrm{HCl}$ & A & 136 & $\begin{array}{l}\mathrm{C}_{24} \mathrm{H}_{32} \mathrm{~N}_{4} \mathrm{OS} \cdot 3 \mathrm{HCl} \\
\cdot \mathrm{H}_{2} \mathrm{O}\end{array}$ & $\begin{array}{r}52.22 \\
(51.98\end{array}$ & $\begin{array}{l}6.76 \\
6.71\end{array}$ & $\begin{array}{l}10.15 \\
10.12\end{array}$ & $\begin{array}{l}5.81 \\
5.93)\end{array}$ & 0.072 \\
\hline $3 \mathbf{a a}$ & $\mathrm{N}\left(\mathrm{CH}_{2} \mathrm{CH}_{2}\right)_{2} \mathrm{~N}\left(\mathrm{CH}_{2}\right)_{5} \mathrm{CH}_{3}$ & Fumarate & A & 128 & $\mathrm{C}_{23} \mathrm{H}_{34} \mathrm{~N}_{4} \mathrm{O}_{5} \mathrm{~S}$ & $\begin{array}{r}57.72 \\
(57.60\end{array}$ & $\begin{array}{l}7.16 \\
7.22\end{array}$ & $\begin{array}{l}11.71 \\
11.61\end{array}$ & $\begin{array}{l}6.70 \\
6.60)\end{array}$ & 0.26 \\
\hline 3ab & $\mathrm{N}\left(\mathrm{CH}_{2} \mathrm{CH}_{2}\right)_{2} \mathrm{~N}\left(\mathrm{CH}_{2}\right)_{6} \mathrm{CH}_{3}$ & Fumarate & A & 153 & $\mathrm{C}_{24} \mathrm{H}_{36} \mathrm{~N}_{4} \mathrm{O}_{5} \mathrm{~S}$ & $\begin{array}{r}58.51 \\
(58.46\end{array}$ & $\begin{array}{l}7.37 \\
7.38\end{array}$ & $\begin{array}{l}11.37 \\
11.28\end{array}$ & $\begin{array}{l}6.51 \\
6.63)\end{array}$ & 0.11 \\
\hline 3ac & $\mathrm{N}\left(\mathrm{CH}_{2} \mathrm{CH}_{2}\right)_{2} \mathrm{~N}\left(\mathrm{CH}_{2}\right)_{7} \mathrm{CH}_{3}$ & Fumarate & A & 135 & $\mathrm{C}_{25} \mathrm{H}_{38} \mathrm{~N}_{4} \mathrm{O}_{5} \mathrm{~S}$ & $\begin{array}{r}59.27 \\
(59.01\end{array}$ & $\begin{array}{l}7.56 \\
7.66\end{array}$ & $\begin{array}{l}11.06 \\
10.95\end{array}$ & $\begin{array}{l}6.33 \\
6.27)\end{array}$ & 0.067 \\
\hline 3ad & $\mathrm{N}\left(\mathrm{CH}_{2} \mathrm{CH}_{2}\right)_{2} \mathrm{~N}\left(\mathrm{CH}_{2}\right)_{9} \mathrm{CH}_{3}$ & $3 \mathrm{HCl}$ & A & 170 & $\begin{array}{l}\mathrm{C}_{23} \mathrm{H}_{37} \mathrm{~N}_{4} \mathrm{OS} \cdot 3 \mathrm{HCl} \\
\cdot \mathrm{H}_{2} \mathrm{O}\end{array}$ & $\begin{array}{r}50.59 \\
(50.50\end{array}$ & $\begin{array}{l}7.94 \\
7.81\end{array}$ & $\begin{array}{l}10.26 \\
10.22\end{array}$ & $\begin{array}{l}5.87 \\
6.07)\end{array}$ & 0.028 \\
\hline 3ae & $\mathrm{N}\left(\mathrm{CH}_{2} \mathrm{CH}_{2}\right)_{2} \mathrm{~N}\left(\mathrm{CH}_{2}\right)_{2} \mathrm{CH}\left(\mathrm{CH}_{3}\right)_{2}$ & $3 \mathrm{HCl}$ & A & 153 & $\begin{array}{l}\mathrm{C}_{18} \mathrm{H}_{28} \mathrm{~N}_{4} \mathrm{OS} \cdot 3 \mathrm{HCl} \\
\cdot 1.7 \mathrm{H}_{2} \mathrm{O}\end{array}$ & $\begin{array}{r}44.26 \\
(44.28\end{array}$ & $\begin{array}{l}7.10 \\
6.97\end{array}$ & $\begin{array}{l}11.47 \\
11.47\end{array}$ & $\begin{array}{l}6.56 \\
6.74)\end{array}$ & 0.16 \\
\hline 3af & $\mathrm{N}\left(\mathrm{CH}_{2} \mathrm{CH}_{2}\right)_{2} \mathrm{CH}\left(\mathrm{CH}_{2}\right)_{2} \mathrm{Ph}$ & $2 \mathrm{HCl}$ & A & 117 & $\begin{array}{l}\mathrm{C}_{22} \mathrm{H}_{27} \mathrm{~N}_{3} \mathrm{OS} \cdot 2 \mathrm{HCl} \\
\cdot 0.8 \mathrm{H}_{2} \mathrm{O}\end{array}$ & $\begin{array}{r}56.36 \\
(56.27\end{array}$ & $\begin{array}{l}6.58 \\
6.55\end{array}$ & $\begin{array}{l}8.96 \\
8.92\end{array}$ & $\begin{array}{l}6.84 \\
6.94)\end{array}$ & 900 \\
\hline $3 a g$ & $\mathrm{~N}\left(\mathrm{CH}_{2} \mathrm{CH}_{2}\right)_{2} \mathrm{CH}\left(\mathrm{CH}_{2}\right)_{3} \mathrm{Ph}$ & $2 \mathrm{HCl}$ & A & 112 & $\begin{array}{l}\mathrm{C}_{23} \mathrm{H}_{29} \mathrm{~N}_{3} \mathrm{OS} \cdot 2 \mathrm{HCl} \\
\cdot 0.4 \mathrm{H}_{2} \mathrm{O}\end{array}$ & $\begin{array}{r}58.07 \\
(58.03\end{array}$ & $\begin{array}{l}6.74 \\
6.64\end{array}$ & $\begin{array}{l}8.83 \\
8.80\end{array}$ & $\begin{array}{l}6.74 \\
6.81)\end{array}$ & 1.9 \\
\hline 3ah & $\mathrm{NHCH}\left(\mathrm{CH}_{2} \mathrm{CH}_{2}\right)_{2} \mathrm{NCH}_{2} \mathrm{Ph}$ & - & $\mathrm{A}$ & 134 & $\mathrm{C}_{21} \mathrm{H}_{26} \mathrm{~N}_{4} \mathrm{OS}$ & $\begin{array}{r}65.94 \\
(65.69\end{array}$ & $\begin{array}{l}6.85 \\
6.83\end{array}$ & $\begin{array}{l}14.65 \\
14.46\end{array}$ & $\begin{array}{l}8.38 \\
8.43)\end{array}$ & 29 \\
\hline & WEB-2086 & & & & & & & & & 0.12 \\
\hline
\end{tabular}


Table 3. PAF-Antagonist Activities of Selected Thiazolidinecarboxamides

\begin{tabular}{|c|c|c|c|c|c|}
\hline \multirow{2}{*}{$\begin{array}{l}\text { Compound } \\
\text { No. }\end{array}$} & \multicolumn{2}{|c|}{$\begin{array}{l}\text { Platelet aggregation inhibition } \\
\qquad \mathrm{IC}_{50}(\mu \mathrm{M})^{a)}\end{array}$} & \multicolumn{3}{|c|}{$\mathrm{ED}_{50}(\mathrm{mg} / \mathrm{kg}$ p.o. $)$} \\
\hline & Rabbit & Human & $\begin{array}{l}\text { Hemoconcentration } \\
\text { in rats }{ }^{b}\end{array}$ & $\begin{array}{l}\text { Vascular permeability } \\
\text { in G. P. } \operatorname{skin}^{c)}\end{array}$ & $\begin{array}{l}\text { PAF-induced death } \\
\text { in mice }{ }^{d)}\end{array}$ \\
\hline 31 & 0.39 & 2.2 & 8.4 & $(8 \%)^{e)}$ & 12.5 \\
\hline $3 n$ & 0.24 & 0.10 & 7.5 & 12.9 & 2.0 \\
\hline $3 \mathbf{x}$ & 0.071 & 0.11 & 0.22 & 2.1 & 0.33 \\
\hline $3 z$ & 0.072 & 1.0 & 1.1 & 4.6 & 3.8 \\
\hline $3 \mathbf{a c}$ & 0.067 & f) & 0.83 & 7.6 & 4.5 \\
\hline 3ad & 0.028 & 0.16 & 3.0 & 13.5 & 2.1 \\
\hline WEB-2086 & 0.12 & 0.11 & 4.2 & 0.37 & 0.57 \\
\hline
\end{tabular}

a) Concentration needed to inhibit PAF (200-400 nM)-induced platelet aggregation in human PRP by $50 \%$. b) Compounds were orally administered $1 \mathrm{~h}$ prior to PAF (1.65 $\mu \mathrm{g} / \mathrm{kg}$ i.v.). c) Compounds were orally administered $1 \mathrm{~h}$ prior to PAF $(5.5 \mathrm{ng} / \mathrm{site}$ i.d.). d) Compounds were orally administered $1 \mathrm{~h}$ prior to PAF $(12.5 \mu \mathrm{g} / \mathrm{kg}$ i.v.). e) Inhibition percent at $30 \mathrm{mg} / \mathrm{kg}$ p.o. f) Not determined.

platelets and have very similar structures.

Next, the compounds were tested for their in vivo activity to inhibit PAF-induced death in mice, hemoconcentration in rats and the increase in vascular permeability in guinea-pigs after oral administration. In general, piperazides exhibited better oral activities than benzyl amides. Among the piperazides, aralkyl piperazides $(\mathbf{3 x}, \mathbf{3 z})$ were more potent than alkyl piperazides (3ac, 3ad). Above all, $3 \mathbf{x}$ showed the best oral activities. This compound was nineteen times and two times more potent than WEB-2086 in rats and mice, respectively, but five times less potent in guinea-pigs. We selected $\mathbf{3 x}$ as a candidate for further evaluation.

Compound 3x inhibited $\left[{ }^{3} \mathrm{H}\right] \mathrm{PAF}$ binding to rabbit platelet membranes with a $\mathrm{p} K_{\mathrm{i}}$ value of 8.90. In an in vivo study, $\mathbf{3 x}$ inhibited the bronchoconstriction induced by PAF $\left(\mathrm{ED}_{50}=1.2 \mathrm{mg} / \mathrm{kg}\right.$ p.o. $)$ in anesthetized guinea-pigs. The compound at doses above $3 \mathrm{mg} / \mathrm{kg}$ p.o. significantly inhibited antigen-induced anaphylactic asthma in conscious guinea-pigs. ${ }^{13)}$

Metabolic study of $\mathbf{3 x}$ revealed that it is metabolized to give piperazide (8) and unsaturated ketone (9) in guinea pigs after oral administration. ${ }^{14)}$ The metabolic pathway appears to be as shown in Chart 3. Compound $\mathbf{3} \mathbf{x}$ was oxidized at the benzyl position to give 3am and then retro-Michael type degradation gave $\mathbf{8}$ and $\mathbf{9}$. Such degradation might lead to a short duration of action, which could be a drawback in clinical use. Thus, to prevent benzylic oxidation, we introduced some substituents at the benzylic position of $\mathbf{3 x}$ and tested these derivatives for PAF-antagonistic activity (Table 4).

The methyl derivative (3ai) was almost as potent as $\mathbf{3 x}$. The introduction of a methoxy (3aj) or a phenyl (3ak) group reduced the activity. The dimethyl derivative (3al) was twice as potent as $\mathbf{3 x}$. The oxo derivative (3am) was also a potent antagonist. We selected 3ai, 3al and 3am for further evaluation. The results are shown in Table 5 .

Compounds 3al and 3am inhibited human and rabbit platelet aggregation at almost the same concentrations. In the in vivo studies, all of the compounds potently inhibited PAF-induced death in mice, hemoconcentration in rats and vascular permeability in guinea-pigs after oral administration. Among them, 3al (YM264) exhibited the best oral activities. YM264 showed $\mathrm{ED}_{50}$ values of 0.30 ,

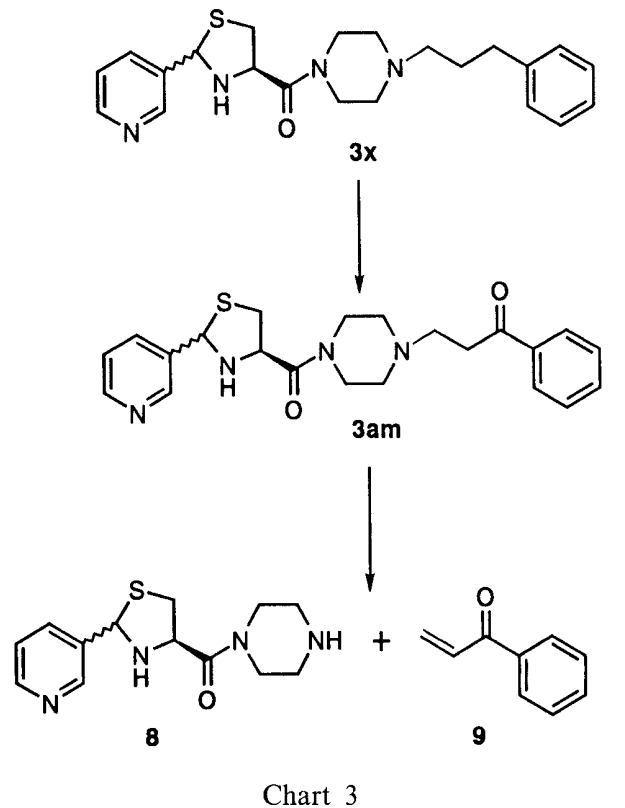

0.49 and $0.19 \mathrm{mg} / \mathrm{kg}$ p.o. in rats, guinea-pigs and mice respectively. These results show that YM264 is a potent and orally active PAF antagonist. The data also suggest that there is little species difference in the anti-PAF action of YM264.

YM264 inhibited $\left[{ }^{3} \mathrm{H}\right] \mathrm{PAF}$ binding to rabbit platelet membrane with a $\mathrm{p} K_{\mathrm{i}}$ value of 8.85 . The $\mathrm{pA}_{2}$ values for YM264 against PAF-induced aggregation in human, rabbit and guinea-pig platelets were $8.68,8.33$ and 8.14 , respectively. YM264, at $10^{-4} \mathrm{M}$, did not exhibit any inhibitory effect on rabbit or human platelet aggregation induced by ADP, collagen, arachidonic acid and epinephrine. ${ }^{15)}$ These results indicate that YM264 is a potent and selective antagonist of PAF.

In the in vivo tests, YM264 inhibited the PAF-induced hypotension in rats with an $\mathrm{ED}_{50}$ value of $0.005 \mathrm{mg} / \mathrm{kg}$ i.v. The inhibitory effect of YM264, after 1 and $3 \mathrm{mg} / \mathrm{kg}$ p.o. persisted for more than $6 \mathrm{~h}$ in the rat hemoconcentration model, indicating that the action of YM264 is relatively long-lasting. ${ }^{15)}$ In an animal model of asthma, the compound at doses above $0.3 \mathrm{mg} / \mathrm{kg}$ p.o. significantly inhibited antigen-induced anaphylactic asthma in conscious guinea pigs. ${ }^{1)}$ YM264 also showed beneficial ef- 
Table 4. Physicochemical Data and PAF-antagonist Activities for Thiazolidinecarboxamides

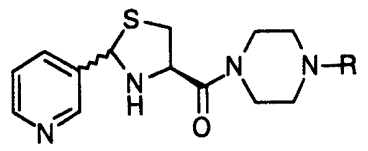

\begin{tabular}{|c|c|c|c|c|c|c|c|c|c|c|}
\hline \multirow{2}{*}{$\begin{array}{l}\text { Compd. } \\
\text { No. }\end{array}$} & \multirow{2}{*}{$\mathrm{R}$} & \multirow{2}{*}{ Salt } & \multirow{2}{*}{ Method } & \multirow{2}{*}{$\mathrm{mp}\left({ }^{\circ} \mathrm{C}\right)$} & \multirow{2}{*}{ Formula } & \multicolumn{4}{|c|}{$\begin{array}{l}\text { Analysis (\%) } \\
\text { Calcd (Found) }\end{array}$} & \multirow{2}{*}{$\begin{array}{c}\text { Platelet } \\
\text { aggregation } \\
\text { inhibition } \\
\mathrm{IC}_{50}(\mu \mathrm{M})\end{array}$} \\
\hline & & & & & & $\mathrm{C}$ & $\mathrm{H}$ & $\mathrm{N}$ & $S$ & \\
\hline 3ai & $\mathrm{CH}_{2} \mathrm{CH}_{2} \mathrm{CH}\left(\mathrm{CH}_{3}\right) \mathrm{Ph}$ & Fumarate & A & $166-168$ & $\mathrm{C}_{27} \mathrm{H}_{34} \mathrm{~N}_{4} \mathrm{O}_{5} \mathrm{~S}$ & $\begin{array}{r}61.58 \\
(61.21\end{array}$ & $\begin{array}{l}6.51 \\
6.45\end{array}$ & $\begin{array}{l}10.64 \\
10.58\end{array}$ & $\begin{array}{l}6.09 \\
6.42)\end{array}$ & 0.064 \\
\hline 3aj & $\mathrm{CH}_{2} \mathrm{CH}_{2} \mathrm{CH}\left(\mathrm{OCH}_{3}\right) \mathrm{Ph}$ & Fumarate & A & $129-132$ & $\mathrm{C}_{27} \mathrm{H}_{34} \mathrm{~N}_{4} \mathrm{O}_{6} \mathrm{~S}$ & $\begin{array}{r}60.41 \\
(60.01\end{array}$ & $\begin{array}{l}6.52 \\
6.39\end{array}$ & $\begin{array}{r}10.06 \\
9.92\end{array}$ & $\begin{array}{l}5.76 \\
5.89)\end{array}$ & 0.31 \\
\hline 3ak & $\mathrm{CH}_{2} \mathrm{CH}_{2} \mathrm{CH}(\mathrm{Ph}) \mathrm{Ph}$ & Fumarate & A & $169-172$ & $\mathrm{C}_{32} \mathrm{H}_{36} \mathrm{~N}_{4} \mathrm{O}_{5} \mathrm{~S}$ & $\begin{array}{r}65.29 \\
(65.55\end{array}$ & $\begin{array}{l}6.16 \\
6.10\end{array}$ & $\begin{array}{l}9.52 \\
9.44\end{array}$ & $\begin{array}{l}5.45 \\
5.70)\end{array}$ & 0.32 \\
\hline 3al & $\mathrm{CH}_{2} \mathrm{CH}_{2} \mathrm{C}\left(\mathrm{CH}_{3}\right)_{2} \mathrm{Ph}$ & Fumarate & A & $172-173$ & $\mathrm{C}_{28} \mathrm{H}_{36} \mathrm{~N}_{4} \mathrm{O}_{5} \mathrm{~S}$ & $\begin{array}{r}62.20 \\
(61.92\end{array}$ & $\begin{array}{l}6.71 \\
6.67\end{array}$ & $\begin{array}{l}10.36 \\
10.17\end{array}$ & $\begin{array}{l}5.93 \\
6.09)\end{array}$ & 0.030 \\
\hline 3am & $\mathrm{CH}_{2} \mathrm{CH}_{2} \mathrm{CH}_{2} \mathrm{COPh}$ & $3 \mathrm{HCl}$ & A & 145 & $\begin{array}{l}\mathrm{C}_{23} \mathrm{H}_{27} \mathrm{~N}_{4} \mathrm{O}_{2} \mathrm{~S} \\
\cdot 3 \mathrm{HCl} \cdot 1.5 \mathrm{H}_{2} \mathrm{O}\end{array}$ & $\begin{array}{r}49.25 \\
(49.40\end{array}$ & $\begin{array}{l}6.11 \\
5.97\end{array}$ & $\begin{array}{l}9.99 \\
9.79\end{array}$ & $\begin{array}{l}5.72 \\
5.92)\end{array}$ & 0.034 \\
\hline
\end{tabular}

Table 5. PAF-antagonist Activities of Selected Thiazolidinecarboxamides

\begin{tabular}{|c|c|c|c|c|c|}
\hline \multirow{2}{*}{$\begin{array}{l}\text { Compound } \\
\text { No. }\end{array}$} & \multicolumn{2}{|c|}{$\begin{array}{l}\text { Platelet aggregation inhibition } \\
\qquad \operatorname{IC}_{50}(\mu \mathrm{M})^{a)}\end{array}$} & \multicolumn{3}{|c|}{$\mathrm{ED}_{50}(\mathrm{mg} / \mathrm{kg}$ p.o. $)$} \\
\hline & Rabbit & Human & $\begin{array}{l}\text { Hemoconcentration } \\
\text { in rats }\end{array}$ & $\begin{array}{l}\text { Vascular permeability } \\
\text { in G. P. skin }\end{array}$ & $\begin{array}{l}\text { PAF-induced death } \\
\text { in mice }\end{array}$ \\
\hline 3ai & 0.064 & e) & 0.14 & 1.1 & 0.73 \\
\hline 3al & 0.030 & 0.030 & 0.30 & 0.49 & 0.19 \\
\hline 3am & 0.034 & 0.027 & 0.19 & 9.0 & 0.4 \\
\hline WEB-2086 & 0.12 & 0.11 & 4.2 & 0.37 & 0.57 \\
\hline
\end{tabular}

a) Concentration needed to inhibit PAF (200-400 nM)-induced platelet aggregation in human PRP by $50 \%$. b) Compounds were orally administered $1 \mathrm{~h}$ prior to PAF $(1.65 \mu \mathrm{g} / \mathrm{kg}$ i.v.). c) Compounds were orally administered $1 \mathrm{~h}$ prior to PAF $(5.5 \mathrm{ng} / \mathrm{site}$ i.d.). $\quad d$ ) Compounds were orally administered $1 \mathrm{~h}$ prior to PAF (12.5 $\mu \mathrm{g} / \mathrm{kg}$ i.v.). Number in parentheses is the number of determinations. e) Not determined.

fects in some models of glomerulonephritis. For example, YM264 inhibited proteinuria and improved hypercholesterolemia and hypoalbuminemia in puromycin aminonucleoside-induced nephropathy in rats. ${ }^{16)}$

In summary, we developed a new series of PAF antagonists, 2-(3-pyridyl)thiazolidine-4-carboxamides. In particular, 3al (YM264) was shown to be a potent and orally active antagonist. Further studies on these compounds are in progress.

\section{Experimental}

Melting points were taken on a Yanaco MP-3 melting point apparatus and are uncorrected. ${ }^{1} \mathrm{H}-\mathrm{NMR}$ were taken with a JEOL FX-90Q, FX100 or GSX-400 spectrometer with tetramethylsilane as an internal standard. Mass spectra were determined on an MS-5970 or JEOL JMSDX300 mass spectrometer. Column chromatography was carried out on silica gel (Wakogel C-200).

Unless otherwise noted, all reagents and solvents obtained from commercial suppliers were used without further purification. In general, organic extract was dried over anhydrous $\mathrm{Na}_{2} \mathrm{SO}_{4}$ or $\mathrm{MgSO}_{4}$ and the solvent was evaporated under reduced pressure. All nonaqueous reactions were performed in dry glassware under an atmosphere of dry Ar.

$N$-tert-Butoxycarbonyl-2-(3-pyridyl)thiazolidine-4-carboxylic Acid (5) Di-tert-butyl dicarbonate $(2.4 \mathrm{~g}, 11 \mathrm{mmol})$ and $10 \mathrm{ml}$ of $1 \mathrm{~N}$ aqueous $\mathrm{NaOH}$ were added to a mixture of $2.1 \mathrm{~g}(10 \mathrm{mmol})$ of 2-(3-pyridyl)thiazolidine-4-carboxylic acid (4), $20 \mathrm{ml}$ of water and $40 \mathrm{ml}$ of dioxane at $4^{\circ} \mathrm{C}$, and the mixture was stirred at room temperature for $30 \mathrm{~min}$, then concentrated. The residue was taken up in $30 \mathrm{ml}$ of water, the $\mathrm{pH}$ was adjusted to 2 by the addition of $0.5 \mathrm{M}$ aqueous citric acid, and the mixture was extracted with ethyl acetate. The extract was washed with water, dried and concentrated, and the residue was recrystallized from ethyl acetate to give $1.0 \mathrm{~g}(3.2 \mathrm{mmol}, 32 \%)$ of $5, \mathrm{mp} 167-169^{\circ} \mathrm{C}$. ${ }^{1} \mathrm{H}-\mathrm{NMR}$ (DMSO-d $\left.d_{6}\right) \delta: 1.12(\mathrm{~s}, 5.4 \mathrm{H}), 1.36(\mathrm{~s}, 3.6 \mathrm{H}), 3.05-3.28(\mathrm{~m}$, $1 \mathrm{H}), 3.48-3.54(\mathrm{~m}, 1 \mathrm{H}), 4.55-4.64(\mathrm{~m}, 0.4 \mathrm{H}), 4.72-4.81(\mathrm{~m}, 0.6 \mathrm{H})$, $6.0 \mathrm{I}(\mathrm{s}, 0.6 \mathrm{H}), 6.18(\mathrm{~s}, 0.4 \mathrm{H}), 7.33-7.42(\mathrm{~m}, 1 \mathrm{H}), 7.98-8.12(\mathrm{~m}, 1 \mathrm{H})$, $8.41-8.51(\mathrm{~m}, 1 \mathrm{H}), 8.73-8.84(\mathrm{~m}, 1 \mathrm{H}), 13.12(\mathrm{br}, 1 \mathrm{H})$; chemical ionization $(\mathrm{CI})$ mass spectrometry $\mathrm{m} / z 311\left(\mathrm{M}^{+}+\mathrm{H}\right)$; Anal. Calcd for $\mathrm{C}_{14} \mathrm{H}_{18} \mathrm{~N}_{2} \mathrm{O}_{4} \mathrm{~S}$ : C, 54.18; H, 5.85; N, 9.03; S, 10.33. Found: C, 54.17; $\mathrm{H}, 5.76 ; \mathrm{N}, 9.06 ; \mathrm{S}, 10.32$.

1,3-Dioxo-5-(3-pyridyl)thiazolidino[3,4-c] oxazolidine Hydrochloride (7) $(\mathrm{COCl})_{2}(0.21 \mathrm{ml})$ was added to a mixture of $5(500 \mathrm{mg}, 1.6 \mathrm{mmol})$, dimethylformamide (DMF) (1 drop) and $\mathrm{CH}_{2} \mathrm{Cl}_{2}(5 \mathrm{ml})$ at $-78^{\circ} \mathrm{C}$ and the mixture was stirred at room temperature for $12 \mathrm{~h}$. The crystals were collected by filtration to give $280 \mathrm{mg}(1.0 \mathrm{mmol}, 64 \%)$ of $7: \mathrm{mp} 170^{\circ} \mathrm{C}$; electron impact (EI) mass spectra $m / z 236\left(\mathrm{M}-\mathrm{HCl}^{+}\right)$; Anal. Calcd for $\mathrm{C}_{10} \mathrm{H}_{9} \mathrm{ClN}_{2} \mathrm{O}_{3} \mathrm{~S}$ : C, 44.04; H, 3.33; N, 10.27; S, 13.00. Found: C, 43.94; H, 3.37; N, 10.24; S, 13.30.

Method A. $N$-Decyl-2-(3-pyridyl)thiazolidine-4-carboxamide (3e) A mixture of $500 \mathrm{mg}(2.4 \mathrm{mmol})$ of $4,370 \mathrm{mg}(2.4 \mathrm{mmol})$ of decylamine, $500 \mathrm{mg}(2.4 \mathrm{mmol})$ of DCC and $500 \mathrm{mg}(3.7 \mathrm{mmol})$ of HOBT in $25 \mathrm{ml}$ of tetrahydrofuran (THF) was stirred overnight at room temperature. The reaction mixture was diluted with ethyl acetate, and the insoluble material was removed by the filtration. The filtrate was washed with aqueous $\mathrm{NaHCO}_{3}$ and a saturated aqueous solution of $\mathrm{NaCl}$, dried and concentrated. The purification of the residue by silica gel column chromatography (eluent; ethyl acetate: methanol $=10: 1$ ) gave $690 \mathrm{mg}$ $(2.0 \mathrm{mmol}, 84 \%)$ of $3 \mathrm{e}: \mathrm{mp} 88^{\circ} \mathrm{C} ;{ }^{1} \mathrm{H}-\mathrm{NMR}\left(\mathrm{CDCl}_{3}\right) \delta: 1.76-1.95(\mathrm{~m}$, $3 \mathrm{H}), 2.10-2.70(\mathrm{~m}, 16 \mathrm{H}), 3.17-3.48(\mathrm{~m}, 3 \mathrm{H}), 3.60-3.76(\mathrm{~m}, 1 \mathrm{H})$, $4.22-4.36(\mathrm{~m}, \mathrm{lH}), 5.42(\mathrm{~s}, 0.9 \mathrm{H}), 5.62(\mathrm{~s}, 0.1 \mathrm{H}), 7.25-7.40(\mathrm{~m}, \mathrm{lH})$, $7.77-7.91(\mathrm{~m}, 1 \mathrm{H}), 8.59(\mathrm{dd}, J=5.0,2.0 \mathrm{~Hz}, 1 \mathrm{H}), 8.74(\mathrm{~d}, J=5.0 \mathrm{~Hz}$ $1 \mathrm{H})$; mass spectrum (EI) $m / z 349\left(\mathrm{M}^{+}+\mathrm{H}\right)$.

Method B. $N$-(4-Benzyloxybenzyl)-2-(3-pyridyl)thiazolidine-4-carbox- 
amide (3j) A solution of $210 \mathrm{mg}(0.99 \mathrm{mmol})$ of 4 and triethylamine $(280 \mu 1,2.0 \mathrm{mmol})$ in $4 \mathrm{ml}$ of DMF was cooled on ice and a solution of DPPA $(270 \mathrm{mg}, 0.98 \mathrm{mmol})$ in $2 \mathrm{ml}$ of DMF and a solution of $220 \mathrm{mg}(0.88 \mathrm{mmol})$ of 4-benzyloxybenzylamine hydrochloride $(220 \mathrm{mg}$, $0.88 \mathrm{mmol}$ ) in $3 \mathrm{ml}$ of DMF were added to it. The mixture was stirred at room temperature for $12 \mathrm{~h}$. After dilution with ethyl acetate, the reaction mixture was washed with a saturated aqueous solution of $\mathrm{NaHCO}_{3}$, water and a saturated aqueous solution of $\mathrm{NaCl}$, dried and concentrated. The residue was recrystallized from 2-propanol to give $160 \mathrm{mg}(0.39 \mathrm{mmol}, 44 \%)$ of $3 \mathrm{j}: \mathrm{mp} 139-140{ }^{\circ} \mathrm{C} ;{ }^{1} \mathrm{H}-\mathrm{NMR}\left(\mathrm{CDCl}_{3}\right) \delta$ $3.30-3.50(\mathrm{~m}, 1 \mathrm{H}), 3.65-3.83(\mathrm{~m}, 1 \mathrm{H}), 4.23-4.25(\mathrm{~m}, 3 \mathrm{H}), 5.06(\mathrm{~s}$ $2 \mathrm{H}), 5.32(\mathrm{~s}, 0.6 \mathrm{H}), 5.44(\mathrm{~s}, 0.4 \mathrm{H}), 6.86-7.02(\mathrm{~m}, 2 \mathrm{H}), 7.11-7.46(\mathrm{~m}$ $8 \mathrm{H}), 7.72-7.84(\mathrm{~m}, 1 \mathrm{H}), 8.56(\mathrm{dd}, J=5.0,2.0 \mathrm{~Hz}, 1 \mathrm{H}), 8.67(\mathrm{~d}, J=$ $2.0 \mathrm{~Hz}, 1 \mathrm{H})$; mass spectrum (FAB) $m / z 406\left(\mathrm{M}^{+}+\mathrm{H}\right)$.

Method C. [2-(3-Pyridyl)thiazolidine-4-carbonyl]-L-methionine Methyl Ester Dihydrochloride (3a) To a solution of $600 \mathrm{mg}(1.9 \mathrm{mmol})$ of $5 \mathrm{in}$ $10 \mathrm{ml}$ of THF, $390 \mathrm{mg}(2.0 \mathrm{mmol})$ of L-methionine methyl ester hydrochloride, $390 \mathrm{mg}(2.9 \mathrm{mmol})$ of $\mathrm{HOBT}, 190 \mathrm{mg}(1.9 \mathrm{mmol})$ of $\mathrm{N}$ methylmorpholine and $440 \mathrm{mg}(2.1 \mathrm{mmol})$ of DCC were added below $4{ }^{\circ} \mathrm{C}$. The mixture was stirred at $4{ }^{\circ} \mathrm{C}$ for $1 \mathrm{~h}$. The precipitate was removed by the filtration and the filtrate was concentrated. The residue was taken up in $50 \mathrm{ml}$ of ethyl acetate, the insoluble material was removed by the filtration and the filtrate was washed with $0.5 \mathrm{M}$ aqueous citric acid, water, $5 \%$ aqueous $\mathrm{NaHCO}_{3}$ and water, dried and concentrated to give $440 \mathrm{mg}$ of [3-tert-butoxycabonyl-2-(3-pyridyl)thiazolidine-4-carbonyl]L-methionine methyl ester. This compound was treated with $5 \mathrm{ml}$ of TFA with ice-water cooling, and the mixture was stirred at room temperature for $2 \mathrm{~h}$. The reaction mixture was concentrated. Ethyl acetate was added to the residue, and the mixture was again concentrated. The residue was purified by silica gel column chromatography (eluent; $\mathrm{CHCl}_{3}$ : metha$\mathrm{nol}=10: 1)$ to give $249 \mathrm{mg}(0.70 \mathrm{mmol}, 36 \%)$ of $3 \mathrm{a}:{ }^{1} \mathrm{H}-\mathrm{NMR}\left(\mathrm{CDCl}_{3}\right)$ $\delta: 1.88-2.27(\mathrm{~m}, 2 \mathrm{H}), 2.06(\mathrm{~s}, 1.2 \mathrm{H}), 2.11(\mathrm{~s}, 1.8 \mathrm{H}), 2.40-2.60(\mathrm{~m}, 2 \mathrm{H})$, $3.31-3.45(\mathrm{~m}, 1 \mathrm{H}), 3.64-6.79(\mathrm{~m}, 1 \mathrm{H}), 3.77(\mathrm{~s}, 3 \mathrm{H}), 4.04-4.07(\mathrm{~m}$, $0.4 \mathrm{H}), 3.36-3.38(\mathrm{~m}, 0.6 \mathrm{H}), 4.67-4.74(\mathrm{~m}, 1 \mathrm{H}), 5.48(\mathrm{~s}, 0.6 \mathrm{H}), 5.65(\mathrm{~s}$, $0.4 \mathrm{H}), 7.30-7.34(\mathrm{~m}, 1 \mathrm{H}), 7.76-7.91(\mathrm{~m}, 1 \mathrm{H}), 8.56-8.59(\mathrm{~m}, 1 \mathrm{H})$, $8.74-8.76(\mathrm{~m}, \mathrm{lH})$; mass spectrum $(\mathrm{EI}) \mathrm{m} / \mathrm{z} 355\left(\mathrm{M}^{+}\right)$.

Method D. 1-(3-Phenylpropyl)-4-[2-(3-pyridyl)thiazolidine-4-carbonyl]piperazine (3x) A solution of 1-(3-phenylpropyl)piperazine (40 mg, $0.20 \mathrm{mmol}$ ) in dimethyl sulfoxide (DMSO) $(0.5 \mathrm{ml})$ was added to a solution of $7(50 \mathrm{mg}, 0.18 \mathrm{mmol})$ in DMSO $(1 \mathrm{ml})$ at room temperature. The mixture was stired at room temperature for $2 \mathrm{~h}$, diluted with ethyl acetate, washed with a saturated aqueous solution of $\mathrm{NaHCO}_{3}$, water and a saturated aqueous solution of $\mathrm{NaCl}$, dried and concentrated to give $70 \mathrm{mg}(0.17 \mathrm{mmol}, 94 \%)$ of $3 \mathbf{x}$. The physical properties of this compound were identical with those of $\mathbf{3 x}$ prepared by method A.

PAF-Induced Platelet Aggregation Rabbit and human PRP were obtained by centrifugation at $270 \times g$ for $10 \mathrm{~min}$. The platelet concentrations were adjusted with platelet-poor plasma to $5 \times 10^{8}$ cells $/ \mathrm{ml}$ for rabbit and to $3 \times 10^{8}$ cells $/ \mathrm{ml}$ for human. Platelet aggregation was measured by the method of Born ${ }^{17)}$ using a Hema Tracer VI (Niko Bioscience, Tokyo, Japan). Various concentrations of the test compounds were added to the PRP 2 min prior to the addition of PAF ( $10 \mathrm{nM}$ for rabbit and 200 $400 \mathrm{nM}$ for human). All experiments were carried out within $4 \mathrm{~h}$ following blood collection to avoid a decrease in the sensitivity of the platelets to the aggregating agent.

PAF-Induced Death in Mice ICR mice, 6 weeks old, were injected with $12.5 \mu \mathrm{g} / \mathrm{kg}$ PAF and the mortality was estimated $24 \mathrm{~h}$ after the administration of PAF. The test compounds were administered orally I h prior to PAF.

PAF-Induced Hemoconcentration Wistar rats, weighing 200 to $336 \mathrm{~g}$, were anesthetized with sodium pentobarbital $(50 \mathrm{mg} / \mathrm{kg}$, i.p.) and the right carotid artery was cannulated for blood collection. Hemoconcentration was determined by measuring the changes of hematocrit values before and $10 \mathrm{~min}$ after the intravenous injection of $1.65 \mu \mathrm{g} / \mathrm{kg}$ PAF. The test compounds were administered orally at $1 \mathrm{~h}$ before the PAF injection.

PAF-Induced Increase in Vascular Permeability The test compounds were administered orally to Hartley guinea-pigs, weighing 290 to $355 \mathrm{~g}$, $1 \mathrm{~h}$ prior to the dorsal intradermal injection of $5.5 \mathrm{ng}$ PAF. At $2 \mathrm{~min}$ prior to PAF injection, $1 \mathrm{ml}$ of saline containing $1.8 \%$ Evans blue was injected intravenously. The animals were then sacrificed 40 min after the injection of PAF. The leaked dye at the site of PAF or vehicle injection was extracted according to the method of Harada et al. ${ }^{18)}$ and the absorbance was measured at $620 \mathrm{~nm}$.

Acknowledgements We are indebted to Drs.K. Murase, M. Takeda, $H$. Ino and G. Kon for helpful discussions and the staff of the Measurement Division in their laboratories for the measurements of NMR and mass spectra and for elemental analyses. We also thank T. Hanazato and M. Akiho for the biological results.

\section{References and Notes}

1) A brief report on a part of this work has appeared. Mase T., Hara H., Nagaoka H., Takahashi T., Suzuki T., Yamada T,. Tomioka K., Abstracts of Papers, The 11 th Symposium on Medicinal Chemistry, Tokushima, 1990, p. 48.

2) Deceased.

3) a) Page C. P., "Asthma: Basic Mechanisms and Clinical Management," ed. by Barns P. J., Rodger I. W., Thomson N. C., Academic Press , London, 1988, pp. 283-304; b) Pinckard R. N., Lundwig J. C., McManus L. M., "Inflammation: Basic Principles and Clinical Correlates," ed. by Gallin J. I., Goldstein I. M., Snyderman R., Raven Press, New York, 1988, pp. 139-167; c) Cooper K., Parry M., "Annual Reports in Medicinal Chemistry," Vol. 24, ed. by Allen R. C., Academic Press, New York, 1989, pp. 81-90, and references therein; $d$ ) Hosford D., Braquet P., "Progress in Medicinal Chemistry," Vol. 27, ed. by Ellis G. P., West G. B., Elsevier Amsterdam, 1990, pp. 325-380.

4) Benveniste J., Le Couedic J. P., Kamoun P., Lancet, i, 344-345 (1975).

5) O'Flaherty J. T., Wykle R. L., Miller C. H., Lewis J. C., Waite M., Bass D. A., McCall C. E., DeChatelet L. R., Am. J. Pathol., 103, 70-79 (1981).

6) Vargaftig B. B., Lefort J., Chignard M. Benveniste J., Eur. J. Pharmacol., 65, 185-192 (1980).

7) Blank M. L., Snyder F. Beyers W., Brooks B., Muirhead D. E., Biochem. Biophys. Res. Commun., 90, 1194-1200 (1979).

8) Vemulapalli S., Chin P. J. S., Barnett A., Hypertension, 6, 489-493 (1984).

9) Vargaftig B. B., Benveniste. J., Trends Pharmacol. Sci., 4, 341-343 (1983).

10) For reviews see: a) Handley D. A., Drugs Future, 13, 137-152 (1988); b) Chang M. N., ibid., 11, 869-875 (1986); c) "Handbook of PAF and PAF Antagonists," ed. by Braquet P., CRC Press: Boca Raton, 1991; d) Koltai M., Hosford D., Guinot P., Esanu A., Braquet P., Drugs, 42, 174-204 (1991); e) Whittaker M., "Current Opinion in Therapeutic Patents 1992," Current Drugs LTD., London, May, pp. 583-623.

11) Sédivy P., Caillard C.G., Floch A., Folliard F., Mondot S., Robaut C., Terlain B., Prostaglandins, 30, 688 (1985).

12) Hife T. F, Natarajan R., Shen C. C., Bembi R., J. Am. Chem. Soc., 113, 3071-3079 (1991).

13) Yamada T., Saito M., Mase T., Hara H., Nagaoka H., Murase K., Tomioka K., Lipid, 26, $1179-1183$ (1991).

14) Unpublished data.

15) Yamada T., Tomioka K., Saito M., Horie M., Mase T., Hara H., Nagaoka H., Arch. Int. Pharmacodyn. Therapie, 308, 123-136 (1990).

16) Yamada T., Tomioka K., Horie M., Sakurai Y., Nagaoka H., Mase T., Biochem. Biophys. Res. Commun., 176, 781—785 (1991).

17) Born G. V. R., J. Physiol., Lond., 162, 67-68 (1962).

18) Harada M., Takeuchi M., Fukao T., Katagiri K., J. Pharm. Pharmacol., 23, 218-219 (1971). 